\title{
Efectos del retardo del asentamiento en las expectativas de vida del briozoo Bugula flabellata (Bryozoa: Gymnolaemata)
}

\author{
Effects of delaying settlement on the life expectancy of the bryozoan Bugula flabellata \\ (Bryozoa: Gymnolaemata)
}

\author{
JUAN M. CANCINO ${ }^{1} \&$ JOSÉ A. GALLARDO $^{2}$ \\ ${ }^{1}$ Departamento de Ecología Costera, Facultad de Ciencias, Universidad Católica de la Santísima Concepción, \\ Casilla 297 Concepción, Chile; e-mail: jcancino@ucsc.cl \\ ${ }^{2}$ Dirección actual: Laboratorio de Genética Cuantitativa, Facultad de Ciencias Agronómicas, Universidad de Chile, \\ Santa Rosa 11315, La Pintana, Santiago, Chile
}

\begin{abstract}
RESUMEN
En presencia de un substrato adecuado las larvas lecitotróficas del briozoo Bugula flabellata se asientan preferentemente durante las primeras seis horas luego de su liberación desde las cámaras de incubación, mientras que un porcentaje marginal $(\approx 1 \%)$ pospone el asentamiento entre 6 y $24 \mathrm{~h}$. En este trabajo se demuestra que las larvas de $B$. flabellata forzadas, por agitación mecánica, a posponer el asentamiento entre 6 y $54 \mathrm{~h}$ reducen significativamente su adecuación biológica. Las larvas mantienen su capacidad para asentarse en un nivel cercano al $95 \%$ durante las primeras 24 h de natación. Posteriormente solo un $30 \%$ de las larvas forzadas a retrasar el asentamiento por 24 a 48 h fueron capaces de asentarse. Más aún, la capacidad para metamorfosear disminuye desde casi un $100 \%$ inicial a menos de un $50 \%$ a partir de las 6 h de retardo del asentamiento. Colonias derivadas de larvas que han sido forzadas a retrasar el asentamiento entre 48-54 h tienen al cabo de 21 días un menor número de zooides $(7,6 \pm 1,88)$ que colonias generadas por larvas que se asentaron entre 1-6 h después de la liberación $(12,7 \pm 1,02)$. Los efectos letales y subletales que genera el retardar el asentamiento larval sobre la capacidad para metamorfosear en $B$. flabellata, concuerdan con el patrón de asentamiento larval temprano descrito anteriormente para esta especie. Los efectos letales y subletales de retrasar el asentamiento en especies con larvas lecitotróficas, podrían ser una importante fuerza selectiva que favorecería la liberación larval en condiciones de bajo movimiento de agua.
\end{abstract}

Palabras clave: liberación larval, natación larval, asentamiento, metamorfosis, Bugula, adecuación biológica.

\begin{abstract}
In the presence of a suitable substratum the lecithotrophic larvae of the bryozoan Bugula flabellata preferentially settle within the first six hours after hatching, whereas a marginal percentage of them $(\approx 1 \%)$ delay settlement between 6 and $24 \mathrm{~h}$ after larval release. In the present study we report that larvae of $B$. flabellata forced, by mechanical water shaking, to delay their settlement from 6 to $57 \mathrm{~h}$, significantly reduced their fitness. Bugula flabellata larvae keep their capacity to settle in a level near to $95 \%$ during the first $24 \mathrm{~h}$ after release. Hereafter only $30 \%$ of the larvae forced to delay settlement for 24 to $48 \mathrm{~h}$ was able to settle. Furthermore, larval metamorphosis quickly decreased from an initial $\approx 100 \%$ value to less than $50 \%$ after 6 $\mathrm{h}$ of a forced delayed settlement. Colonies generated by larvae forced to delay settlement for $48-54 \mathrm{~h}$ generated colonies that after 21 days of growth had in average a smaller number of zooids than colonies generated by larvae forced to swim for only $1-6 \mathrm{~h}(7.6 \pm 1.88$ and $12.7 \pm 1.02$, respectively). The lethal and sublethal effects generated by delaying larval settlement on the metamorphosis success of $B$. flabellata correlate well with the larval behaviour of settling soon after release. The lethal and sublethal effects of delaying settlement in species producing lecithotrophic larvae are likely to be a mayor selective force favouring larval release in low water movement conditions.
\end{abstract}

Key words: larval release, larval swimming, settlement, metamorphosis, Bugula, fitness. 


\section{INTRODUCCIÓN}

La mayoría de los briozoos produce larvas lecitotróficas, que no se alimentan durante su fase de vida libre, y las que son liberadas durante el día, en respuesta a la luz (Ryland 1960). Esta liberación ocurre al amanecer o siguiendo un patrón dependiendo de las fluctuaciones del nivel de luz en el agua (Cancino et al. 1991, 1992, 1994). Tal patrón de liberación se ha relacionado con el uso de la luz como un factor de orientación en el asentamiento larval (Cancino et al. 1994, Cancino \& Gallardo 2000). Como en otras especies de invertebrados que producen larvas lecitotróficas (Olson 1983; Pechenik \& Cerruli 1991, Marshall et al. 2003) el asentamiento larval de briozoos ocurre generalmente dentro de unas pocas horas desde la liberación larval (Orellana \& Cancino 1991; Cancino et al. 1994, Cancino \& Gallardo 2000). El asentamiento puede ocurrir tanto en substratos naturales adecuados (Ryland 1974) como en substratos artificiales acondicionado en agua de mar, en los que se haya desarrollado una película microbiana (Cancino \& Hughes 1988). Sin embargo, el asentamiento en briozoos con larvas lecitotróficas puede ser pospuesto por varias horas en las siguientes circunstancias: (a) falta de un substrato adecuado (Orellana \& Cancino 1991, Cancino \& Gallardo 2000); (b) en condiciones de un fuerte movimiento del agua (Orellana \& Cancino 1991, Quian et al. 1999); (c) alta intensidad luminosa (Wendt 1996, 1998); o (d) ante la presencia de competidores (Chia \& Young 1981). Algunas de estas causas y otras como baja temperatura o apiñamiento también pueden retrasar el asentamiento en otras especies de invertebrados (Pechenik \& Cerruli 1991, Pechenik et al. 1993). La capacidad de una larva para posponer el asentamiento debería promover el asentamiento en lugares más adecuados para la vida futura (Hulbut 1991). Sin embargo, en larvas lecitotróficas la habilidad de retrasar el asentamiento está fuertemente limitada por las reservas energéticas que estas posean, generalmente existe un umbral de tiempo para posponer el asentamiento luego del cual se pueden producir fuertes compromisos sobre atributos que afectan la adecuación biológica (Marshall et al. 2003). En briozoos, tales compromisos incluyen reducir la probabilidad de asentarse y metamorfosear (Orellana \& Cancino 1991, Hunter \& Fusetani 1996, Orellana et al. 1996); aumentar el tiempo necesario para completar la metamorfosis (Wendt 1996), y/o disminuir significantemente el crecimiento colonial, lo que se ha demostrado tanto en condiciones de laboratorio (Woolla- cott et al. 1989, Orellana \& Cancino 1991) como en el ambiente natural (Wendt 1998). Como consecuencia de lo anterior se reduce la probabilidad de supervivencia, así larvas que no se asientan o que habiéndose asentado no metamorfosean, son larvas que mueren sin dar origen a un juvenil, lo que equivale a una adecuación biológica con valor cero. Mientras que larvas que tardan en metamorfosear o que generan zooides a una baja tasa comprometen no solo el crecimiento colonial inicial sino que el crecimiento y fecundidad futura, esto debido a que en briozoos el crecimiento inicial es exponencial y la fecundidad es dependiente del tamaño colonial (Cancino \& Hughes 1988).

Bugula flabellata (Thompson 1847) es un briozoo arborescente que vive en ambientes costeros, en zonas no expuestas directamente al oleaje y de moderada intensidad luminosa. Usualmente se le encuentra adherido a otras especies de briozoos, sobre algas, o sobre substratos artificiales como boyas o cuerdas. A pesar de tener una amplia distribución (Ryland 1967, López et al. 1996, Moyano 1996) su ecología larval es poco conocida (Dyrynda \& Ryland 1982). Recientemente, Cancino \& Gallardo (2000) describieron que colonias de $B$. flabellata mantenidas en laboratorio producen una gran cantidad de larvas lecitotróficas, las cuales, en presencia de un sustrato artificial acondicionado en agua de mar, se asientan preferentemente $(\approx 90 \%)$ durante las primeras $6 \mathrm{~h}$ desde la liberación larval, con un máximo asentamiento a las 2,5 h. Un porcentaje marginal $(\approx 1 \%)$ pospone el asentamiento entre 6 y $24 \mathrm{~h}$, mientras que el 9 $\%$ restante no se asentó sobre el sustrato ofrecido. Sobre la base de estos antecedentes, en el presente trabajo nosotros examinamos las consecuencias de retardar el asentamiento sobre: (1) la capacidad para asentarse sobre un substrato previamente acondicionado, (2) la capacidad para metamorfosear y generar una ancéstrula viable, y (3) el crecimiento colonial temprano. Nosotros predecimos que restricciones sobre uno o más de estos componentes deberían ser concordantes con el patrón de asentamiento temprano descrito para esta especie.

\section{MATERIALES Y MÉTODOS}

\section{Recolección de las larvas}

Durante febrero de 1997 se recolectaron colonias sexualmente maduras de $B$. flabellata de substratos artificiales (debajo de boyas) localizados en medio de la bahía de Concepción, 
Chile central $\left(36^{\circ} 40^{\prime}\right.$ S, $73^{\circ} 05^{\prime}$ W). Posteriormente se trasladaron las colonias al Laboratorio Costero Lenga de la Universidad Católica de la Santísima Concepción ubicado en la bahía San Vicente. Las colonias se mantuvieron en agua de mar circulante sin filtrar a una temperatura entre $12-15{ }^{\circ} \mathrm{C}$, y sin entregar una dosis adicional de alimento (microalgas). Para la obtención de las larvas, las colonias se trasladaron a una cámara de cultivo diseñada para este propósito (Cancino et al. 1991). Esta cámara estaba provista de un recipiente de $5 \mathrm{~L}$ en el que se ubican las colonias, y a través de la cual se hacía circular agua de mar mediante una entrada en su base y una salida en su extremo superior. Las cámaras se mantuvieron en oscuridad total por $16 \mathrm{~h}$ y luego fueron expuestas a luz natural para inducir la liberación larval (Cancino \& Gallardo 2000), lo cual ocurrió durante las primeras horas de la mañana. El agua que fluía a través de la cámara arrastraba las larvas liberadas hasta un recipiente de $300 \mathrm{~mL}$ el cual en su extremo inferior tenía un tamiz de $60 \mu \mathrm{m}$ donde las larvas quedaban retenidas. Las larvas se recolectaron cada 15 minutos intercambiando un recipiente por otro y luego se trasladaron a frascos de cultivo independientes para retrasar el asentamiento como se explica mas abajo. En general las larvas utilizadas corresponden a aquellas liberadas durante la segunda hora desde que las colonias fueron expuestas a la luz y cuando el número de larvas obtenidas durante 15 minutos de recolección fueron suficientes para realizar un experimento $(\approx 100$ larvas $)$.

\section{Retardo del asentamiento y probabilidad de asentamiento y metamorfosis}

Para retrasar el asentamiento se colocaron las larvas obtenidas en cada intervalo de recolección en frascos de vidrio independientes $(400$ $\mathrm{mL}$ ). Estos se lavaron previamente con una solución diluida de ácido clorhídrico que previene en parte el asentamiento de las larvas sobre su superficie. Adicionalmente se agitó el agua en cada frasco por medio de paletas plásticas adheridas a un aparato mecánico que producía un movimiento pendular a una velocidad de 1,82 \pm $0,15 \mathrm{~cm} \mathrm{~s}^{-1}$. Este movimiento generaba una fuerte turbulencia al interior de los frascos, impidiendo el asentamiento larval en tales recipientes. Luego de permanecer en estas condiciones durante $6,12,24,48$ y $54 \mathrm{~h}$ (tiempo de retardo del asentamiento) se trasladaron grupos de 11-30 larvas ( $\mathrm{n}=2$ para cada período, total 180 larvas) a placas Petri para evaluar la capacidad de asentamiento. Como grupo control se trasladó larvas directamente del recipiente de recolección a las placas de asentamiento $(0 \mathrm{~h}$ de retardo del asentamiento). Las placas de asentamiento ( $5 \mathrm{~cm}$ de diámetro) contenían $10 \mathrm{~mL}$ de agua de mar filtrada $(0,45 \mu \mathrm{m})$ y habían sido previamente acondicionadas para el asentamiento manteniéndolas durante una semana en agua de mar circulante. El asentamiento se registró luego de $3 \mathrm{~h}$ de haberse puesto las larvas en contacto con el substrato, por lo tanto los tiempos experimentales de retardo del asentamiento fluctuaron entre los siguientes intervalos 0-3, 6-9, 12-15, 24-27, 48-51 y 54-57 h. El número de larvas asentadas se registró con la ayuda de una lupa binocular Nikon, demorando cada registro menos de $3 \mathrm{~min}$ por placa. Una larva se clasificó como asentada si permaneció adherida al substrato cuando se hizo fluir agua sobre ella varias veces con una pipeta Pasteur, las larvas no asentadas fueron desechadas. Para evaluar el éxito en la metamorfosis todas las placas con las larvas asentadas, correspondientes a los distintos tiempos de asentamiento, se trasladaron a una misma cámara de cultivo de 4 $\mathrm{L}$ de capacidad. Estas se mantuvieron a $12 \pm 1$ ${ }^{\circ} \mathrm{C}$ y con burbujeo de aire constante. Diariamente se cambió el agua y se adicionaron 10.000 cel $\mathrm{mL}^{-1}$ de la microalga Rhymomonas sp. para favorecer una temprana alimentación. Al cabo de una semana se determinó el éxito en alcanzar la metamorfosis, calculando la proporción de larvas asentadas que dieron origen a una ancéstrula (a saber el primer zooide de la futura colonia). Se realizó un análisis de independencia $(G)$ con el porcentaje promedio de las dos réplicas para poner a prueba si la capacidad para asentarse y el éxito en la metamorfosis son independientes del tiempo de retardo del asentamiento (Sokal \& Rohlf 1981).

\section{Retardo del asentamiento y crecimiento colonial}

Para investigar el efecto del retardo del asentamiento sobre el crecimiento colonial temprano se procedió a generar cuatro grupos de larvas de acuerdo a los siguientes tiempos de retardo del asentamiento: 1-6 h (grupo 1), 12-18 h (grupo 2), 24-30 h (grupo 3) y de 48-54 h (grupo 4). En total se cultivaron 37 colonias durante 3 semanas en cámaras plásticas de $500 \mathrm{~mL}$, 13 en el grupo 1, 12 en el grupo 2, y 6 colonias en el grupo 3 y 4 . Ellas fueron alimentadas cada dos días con 20.000 cel $\mathrm{mL}^{-1}$ de la microalga Rhymomonas sp. El crecimiento se evaluó semanalmente, contando el número de zooides en cada colonia. Para analizar el efecto del retardo del asentamiento sobre el crecimiento colonial se utilizó un análisis de varianza (AN- 
DEVA), previa transformación logarítmica de los datos (Sokal \& Rohlf 1981).

\section{RESULTADOS}

Las larvas de Bugula flabellata forzadas a retrasar el asentamiento mediante agitación constante y en ausencia de un substrato adecuado, mantienen una alta capacidad para asentarse durante las primeras $24 \mathrm{~h}$ después de la liberación larval (Fig. 1). Durante este periodo más del $95 \%$ de estas larvas fueron capaces de asentarse cuando se ofreció un substrato artificial. Posteriormente una alta proporción de las larvas pierde su capacidad para asentarse sobre el sustrato artificial (Fig. 1), aun cuando en los frascos usados para mantener las larvas por los periodos de retardo más largo se observaron varias larvas asentadas unas sobre otras. Al cabo de $54 \mathrm{~h}$ de la liberación larval el asentamiento se reduce significantemente a solo un $30 \%$ en promedio $(\mathrm{G}=81,2 ; \mathrm{gl}=1 ; \mathrm{P}<0,05)$.

La capacidad para metamorfosear se reduce más tempranamente luego de la liberación lar- val que la capacidad para asentarse (Fig.2). Al cabo de seis horas solo el $50 \%$ de las larvas mantiene la capacidad para metamorfosear y esta capacidad se reduce más aún entre 12 y 54 $\mathrm{h}$ desde la liberación (20\% en promedio). Tal disminución en la capacidad para metamorfosear en función del tiempo de retardo del asentamiento fue estadísticamente significativa $(\mathrm{G}=$ $22,2 ; \mathrm{gl}=1 ; \mathrm{P}<0,05)$. Luego de la metamorfosis casi el $100 \%$ de las ancéstrulas fue capaz de generar una colonia.

El retardo del asentamiento no tuvo efectos significativos en el tamaño colonial durante los primeros 14 días de crecimiento, sin embargo al cabo de 21 días existe una tendencia a que las colonias generadas a partir de larvas forzadas a retardar el asentamiento entre 1-30 h tuvieran en promedio el doble del número de zooides que aquellas generadas por larvas asentadas luego de 48-54 h (Fig. 3). Se detectaron diferencias estadísticamente significativas en el tamaño colonial exclusivamente entre los grupos que retardaron el asentamiento 1-6 h (grupo 1) versus $48-54 \mathrm{~h}$ (grupo 4) $\left(\mathrm{F}_{1,16}=8,42 ; \mathrm{P}<0.05\right)$.

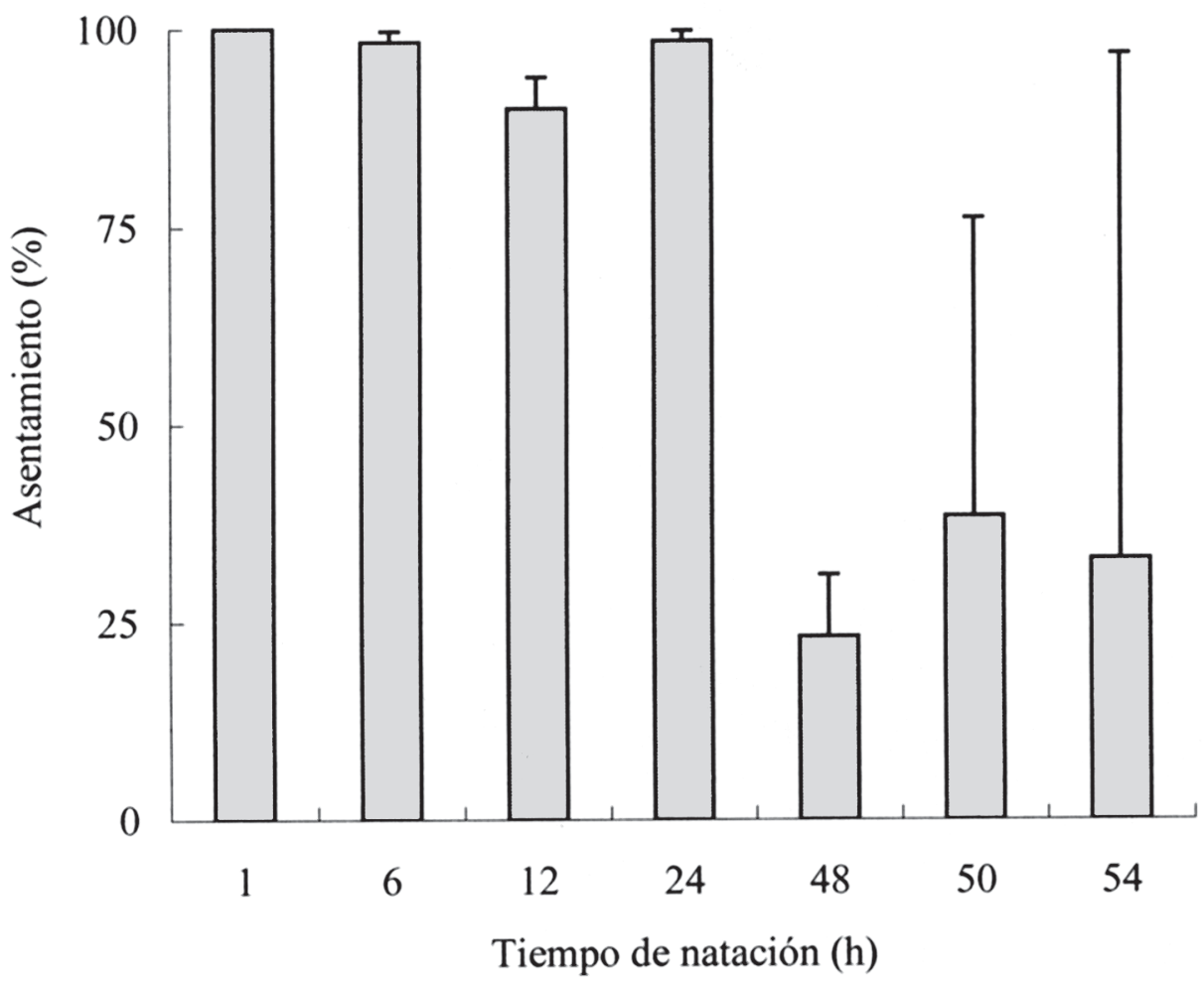

Fig. 1: Porcentaje de asentamiento larval de B. flabellata sobre un substrato acondicionado en agua de mar, en función del tiempo de retardo del asentamiento $(\mathrm{n}=158$ larvas). Se grafica el promedio y el error estándar luego de una transformación angular $(\mathrm{n}=2)$.

Percentage of larval settlement on $B$. flabellata $(n=158$ larvae) as a function of time the larvae were forced to delay settlement before the substratum for settlement was offered. The bars represent the average of two replicates ( \pm 1 SE) calculated after angular transformation. 


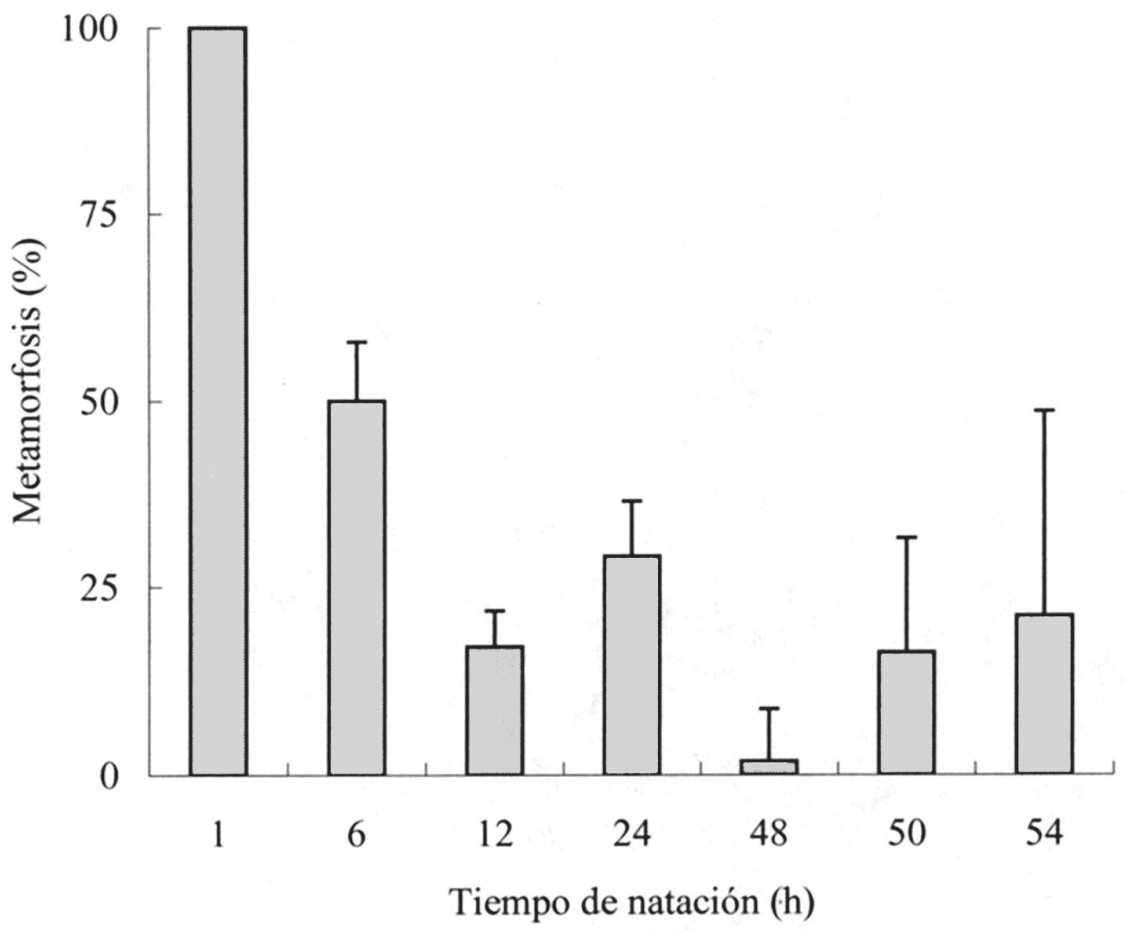

Fig. 2: Porcentaje de larvas de B. flabellata que metamorfosean sobre un substrato acondicionado en agua de mar, en función del tiempo de retardo del asentamiento ( $n=84$ larvas). Se grafica el promedio y el error estándar luego de una transformación angular $(\mathrm{n}=2)$.

Percentage of larval metamorphosis on $B$. flabellata ( $\mathrm{n}=84$ larvae) as a function of time the larvae were forced to delay settlement before the substratum for settlement was offered. Bars represent the average of two replicates $( \pm 1$ SE), calculated after angular transformation.

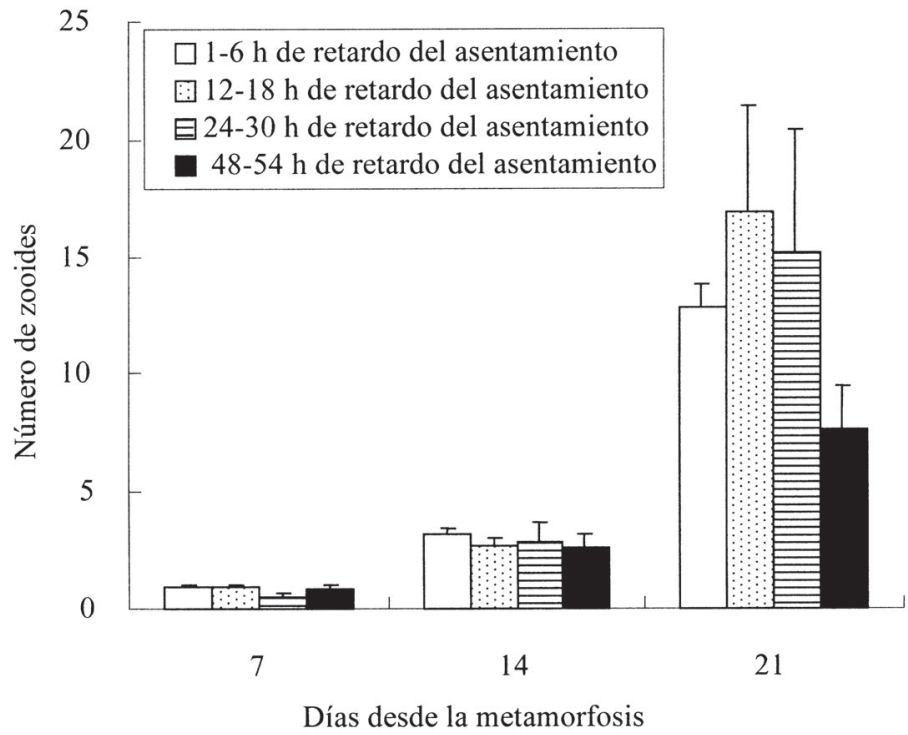

Fig. 3: Tamaño de las colonias de B. flabellata derivadas de larvas que fueron forzadas a retrasar el asentamiento entre 1 y $57 \mathrm{~h}$. Se grafica el promedio y el error estándar; $n=6-13$ colonias por intervalo de natación.

Colonial size on B. flabellata at different times after settlement, as a function of the time the larvae were forced to delay settlement before the substratum for settlement was offered. Values given are mean $\pm 1 \mathrm{SE} ; \mathrm{n}=6-13$ per swimming interval. 


\section{DISCUSIÓN}

En concordancia con lo esperado, el retardo del asentamiento larval por más de $6 \mathrm{~h}$ reduce las expectativas de vida temprana en Bugula flabellata. Todos los componentes examinados fueron afectados negativamente, aunque en distinta magnitud y a distintos tiempos post liberación larval. La metamorfosis fue la variable más severa y tempranamente afectada (6-9 h), mientras que la capacidad para asentarse y el crecimiento colonial fueron menos afectados y solo después de 24 y 48 h de retardo del asentamiento, respectivamente.

\section{EFECTOS LETALES}

\section{Metamorfosis}

De manera similar a lo que ocurre en otras especies de briozoos, esta variable fue la más afectada por el retardo del asentamiento, tanto que $50 \%$ de las larvas fue incapaz de metamorfosear cuando fueron forzadas a nadar por más 6 h. Hunter et al. (1999) describen que en $B$. neritina la capacidad para metamorfosear es el primer y mayor componente afectado cuando las larvas son forzadas a nadar por hasta 8 h. En comparación con otras especies del mismo género $B$. flabellata pierde más temprano la capacidad para metamorfosear. Wendt $(1996,1998)$ determinó que el tiempo medio en que el $50 \%$ de las larvas pierde la capacidad para metamorfosear fue de 14,16 y 20 h para $B$. stolonifera, $B$. simplex y $B$. neritina, respectivamente. Nuestros datos indican que en $B$. flabellata este valor se obtiene entre 6 a 9 horas desde la liberación larval. Los efectos sobre la capacidad para metamorfosis concuerdan con el patrón de asentamiento larval descrito por Cancino \& Gallardo (2000) y en el cual más del $90 \%$ de las larvas de $B$. flabellata se asientan durante las primeras $6 \mathrm{~h}$ desde la liberación.

\section{Asentamiento}

Al contrario de la metamorfosis este componente fue débilmente afectado durante las primeras $24 \mathrm{~h}$ de natación. Pensamos que esto puede explicarse por los costos metabólicos implicados en cada proceso. En otras especies de Bugula Wendt (2000) demuestra que el costo energético de la natación es 2 a 4 veces menor que el costo involucrado en completar la metamorfosis. Por ejemplo. B stolonifera necesita un $52 \%$ de sus reservas endógenas para completar la metamorfosis, mientras que al cabo de 16 h de natación las larvas solo han consumido un $26 \%$ de sus reservas. Así, las reservas energéticas disponibles luego de $24 \mathrm{~h}$ de natación serían suficientes para permitir el asentamiento pero insuficientes para completar la metamorfosis. Una forma de compensar el gasto producido por la natación podría ser reducir la velocidad de natación. Nosotros observamos que las larvas de B. flabellata reducen cualitativamente su velocidad de natación luego de 24 h desde la liberación, una conducta similar ha sido observada en Celleporella hyalina por Orellana \& Cancino (1991). Más estudios son necesarios para evaluar el gasto energético en función del tiempo desde la liberación larval. Sobre la base de esto nosotros predecimos que tanto la velocidad de natación y por ende la energía consumida deben disminuir cuando se retrasa el asentamiento, hipótesis aún no sometida a prueba.

El asentamiento sobre otras larvas es un fenómeno nuevo que no había sido descrito para otras especies de briozoos, sin embargo lo más probable es que este fenómeno sea un artefacto del experimento, pues es poco probable que se genere una colonia en estas condiciones. Luego de $24 \mathrm{~h}$ de natación la mayor parte de las larvas que se mantenía nadando en los frascos de retardo del asentamiento se asentaron sobre otras larvas vivas o muertas, constituyendo estas, aparentemente, el único substrato disponible atractivo para el asentamiento.

\section{EFECTOS SUBLETALES SOBRE EL CRECIMIENTO}

Como en otras especies de briozoos el retardo en el asentamiento produce efectos subletales en el crecimiento colonial. En $B$. flabellata luego de $48 \mathrm{~h}$ de natación los efectos sobre el crecimiento son significativos, reduciéndose el número de zooides en un $40 \%$, lo cual es similar a lo registrado por Wendt (1998) con colonias de $B$. neritina mantenidas en condiciones naturales. Este autor determinó que colonias derivadas de larvas que nadaron por $24 \mathrm{~h}$ son un $35 \%$ más pequeñas que las colonias derivadas de larvas que nadaron solo por $1 \mathrm{~h}$. En otras especies de briozoos un menor crecimiento ha sido explicado por dos factores (Wendt 1996), a saber: (a) el proceso de metamorfosis es más lento en larvas que retrasan el asentamiento, retrasando el tiempo de inicio de la alimentación, o (b) las colonias generadas por larvas que han retrasado el asentamiento consumen menos alimento debido a que la corona tentacular (lofóforo) de sus zooides es más pequeña. 
¿Pueden los efectos letales y subletales de retrasar el asentamiento ser una fuerza selectiva sobre el patrón de liberación larval?

Recientemente se ha descrito que las larvas lecitotróficas de invertebrados marinos se liberan preferentemente en condiciones de bajo flujo de agua (Welch et al. 2000). Ellos sugieren que esto es una forma de retener a la progenie en un lugar adecuado para el asentamiento. Sin embargo, nosotros proponemos una explicación alternativa, esta es que la liberación larval en condiciones de bajo flujo de agua debería producirse como una forma de evitar los efectos perjudiciales de retrasar el asentamiento. En este sentido, existen al menos dos fuerzas selectivas que nos hacen pensar que las larvas de Bugula, y de otros invertebrados con larvas lecitotróficas, deberían ser liberadas en períodos de bajo movimiento de agua en forma superpuesta a los patrones clásicos de liberación larval ya conocidos (i.e., ritmos circadianos), estas son: (1) en varias especies de invertebrados las larvas son incapaces de asentarse cuando el flujo o el movimiento del agua es elevado (Orellana \& Cancino 1991, Wendt 1996, 1998, Qian et al. 1999, 2000); (2) los costos y efectos perjudiciales de retrasar el asentamiento pueden ser muy altos, como se ha demostrado para distintas especies de briozoos (Woollacott et al. 1989, Orellana \& Cancino 1991, Hunter \& Fusetani 1996, Orellana et al. 1996, Wendt 1996, Hunter et al. 1999, Wendt 1998, y presente estudio), de poliquetos (Pechenik \& Cerruli 1991), de cirripedios (Pechenik et al 1993) y de ascidias (Marshall et al. 2003)

\section{AGRADECIMIENTOS}

Agradecemos al Teniente de la Armada de Chile Sr. Rubén Rojas por la ayuda recibida en la recolección de las colonias de briozoos. Esta investigación fue financiada por el proyecto Fondecyt 1940-233 a JMC.

\section{LITERATURA CITADA}

CANCINO JM \& RN HUGHES (1988) The zooidal polymorphism and astogeny of Celleporella hyalina (L). Journal of Zoology London 215: 167-181.

CANCINO JM, RN HUGHES \& C RAMÍREZ (1991) Environmental cues and the phasing of larval release in the bryozoan Celleporella hyalina (L). Proceedings of the Royal Society, London B 246: $39-45$.

CANCINO JM, MC ORELLANA, R MUÑOZ \& RN HUGHES (1992) Ciclo diario de liberación larval en dos especies de Briozoos. Revista de Biología Marina 27: 213-223.
CANCINO JM, R HUGHES \& MC ORELLANA (1994) A comparative study of larval release in bryozoans. En: Hayward P, J Ryland \& P Taylor (eds) Biology and Paleobiology of Bryozoans: 41-45. Olsen \& Olsen, Fredensborg, Denmark.

CANCINO JM \& JA GALLARDO (2000) Effects of light on bryozoan larval settlement. En: Herrera CA \& J Jackson (eds) Proceedings of the $11^{\text {th }}$ International Bryozoology Association Conference:168-175. Smithsonian Tropical Research Institute, Balboa, Republic of Panama.

DYRYNDA EJ \& JS RYLAND (1982) Reproductive strategies and life histories in the cheilostome marine bryozoans Chartella papyracea and Bugula flabellata. Marine Biology 71: 241-256.

HUNTER E \& N FUSETANI (1996) Studies on the effects of larval swimming time on settlement, metamorphosis and post-larval development of Bugula neritina (Cheilostomatida). En: Gordon DP, AM Smith \& JA Grant-Mackie (eds) Bryozoans in Space and Time: 139-148. National Institute of Water and Atmospheric Research Ltd, Wellington, New Zealand.

HUNTER E, K SHIMIZU \& N FUSETANI (1999) Role of protein in larval swimming and metamorphosis of Bugula neritina (Bryozoa: Cheilostomatida). Marine Biology 133: 701-707.

HUNTER E, K OKANO, Y TOMONO \& N FUSETANI (1998) Functional partitioning of energy reserves by larvae of the marine bryozooan Bugula neritina (L). Journal of Experimental Biology 201: 28572865.

HURLBUT CJ (1991) Larval substratum selection and postsettlement mortality as determinants of the distribution of two bryozoans. Journal Experimental Marine Biology and Ecology 147: 103-120.

LÓPEZ C \& J GARCÍA-GÓMEZ (1994) Zoogeographical study of the Cheilostomatida from the straits of Gibraltar. En: Hayward P, J Ryland \& P Taylor (eds) Biology and Paleobiology of Bryozoans: 107-112. Olsen \& Olsen, Fredensborg, Denmark.

MARSHALL DJ, JA PECHENIK \& MJ KEOUGH (2003) Larval activity levels and delayed metamorphosis affect post-larval performance in the colonial ascidian Diplosoma listerianum. Marine Ecology Progress Series 246: 153-162.

MOYANO H (1996) Holocene bryozoan links between Australia, New Zealand, southern America, and Antarctica - a preliminary evaluation. En: Gordon DP, AM Smith \& JA Grant-Mackie (eds) Bryozoans in Space and Time: 207-219. National Institute of Water and Atmospheric Research Ltd, Wellington, New Zealand.

OLSON RR (1983) Ascidian-Prochloron symbiosis: the role of larval photoadaptations in midday larval release and settlement. Biological Bulletin 165: 221-240.

ORELLANA MC \& JM CANCINO (1991) Effects of delaying settlement on metamorphosis and early colonial growth in Celleporella hyalina (Bryozoa: Cheilostomata). En: Bigey FP (ed) Bryozoaries actuels et fossiles: bryozoa living and fossil: 309316. Bulletin de la Société des Sciences Naturelles de 1'Ouest de la France, Nantes, France.

ORELLANA MC, JM CANCINO \& RN HUGHES (1996). Is settlement in lecithotrophic bryozoan larvae constrained by energy reserves?. En: Gordon DP, AM Smith \& JA Grant-Mackie (eds) Bryozoans in Space and Time: 221-226. National Institute of Water and Atmospheric Research Ltd, Wellington, New Zealand. 
PECHENIK JA \& TR CERULLI (1991) Influence of delayed metamorphosis on survival, growth, and reproduction of the marine polychaete Capitella sp. I. Journal Experimental Marine Biology and Ecology: 17-27.

PECHENIK JA, D RITTSCHOF \& AR SCHMIDT (1993) Influence of delayed metamorphosis on survival and growth of juvenile barnacles Balanus amphitrite. Marine Biology: 115:287-294.

QIAN PY, D RITTSCHOF, B SREEDHAR \& FS CHIA (1999) Macrofouling in unidirectional flow: miniature pipes as experimental models for studying the effects of hydrodynamics on invertebrate larval settlement. Marine Ecology Progress Series 191: 141-151.

QIAN PY, D RITTSCHOF \& B SREEDHAR (2000) Macrofouling in unidirectional flow: miniature pipes as experimental models for studying the interaction of flow and surface characteristics on the attachment of barnacle, bryozoan and polychaete larvae. Marine Ecology Progress Series 207: 109-121.

RYLAND JS (1960) Experiments on the influence of light on the behaviour of polyzoan larvae. Journal of Experimental Biology 37: 783-800.

RYLAND JS (1967) Polyzoa. Oceanography and Marine Biology Annual Review 5: 343-369.

RYLAND JS (1974) Behaviour, settlement and metamorphosis of bryozoan larvae: a review. Thalassia Jugoslavica 10: 239-262.
SOKAL R \& F ROHLF (1981) Biometry. Second Edition. WH Freeman and Co., San Francisco, California, USA. $859 \mathrm{pp}$

WELCH JM, LJ WALTERS \& CM YOUNG (2000) Effects of flow on larval release behavior of the bryozoan Bugula neritina. American Zoologist 40: 1254.

WENDT D (1996) Effects of larval swimming duration on success of metamorphosis and size of the ancestrular lophophore in Bugula neritina (Bryozoa). Biological Bulletin 191: 224-233.

WENDT D (1998) Effects of larval swimming duration on growth and reproduction of Bugula neritina (Bryozoa) under field condition. Biological Bulletin 195: 126-135.

WENDT D (2000) Energetics of larval swimming and metamorphosis in four species of Bugula (Bryozoa). Biological Bulletin 198: 346-356.

WOOLLACOTT RM, JA PECHENIK \& KM IMBALZANO (1989) Effects of duration of larval swimming period on early colony development in Bugula stolonifera (Bryozoa: Cheilostomata). Marine Biology 102: 57-63.

YOUNG CM \& FS CHIA (1981) Laboratory evidence for delay of larval settlement in response to a dominant competitor. International Journal of Invertebrate Reproduction 3: 221-226. 